\title{
Structure, Spatial and Temporal Distribution of the Culex pipiens Complex in Shanghai, China
}

\author{
Qiang Gao ${ }^{1,2,3}$, Chenglong Xiong ${ }^{1,2}$, Fei Su ${ }^{3}$, Hui Cao ${ }^{3}$, Jianjun Zhou ${ }^{3, *}$ and Qingwu Jiang ${ }^{2, *}$ \\ 1 Department of Public Health Microbiology, School of Public Health, Fudan University, \\ Shanghai 232000, China; gaoqiang110209@163.com (Q.G.); xiongchenglong@fudan.edu.cn (C.X.) \\ 2 Key Laboratory of Public Health Safety, Ministry of Education, Fudan University, Shanghai 232000, China \\ 3 Department of Vectors Prevention, Center for Disease Control \& Prevention, Huangpu District, \\ Shanghai 232000, China; sufei@hpcdc.sh.cn (F.S.); caohui@hpcdc.sh.cn (H.C.) \\ * Correspondence: zhoujianjun309@163.com (J.Z.); jiangqw@fudan.edu.cn (Q.J.); \\ Tel.: +86-21-5302-3812 (J.Z.); +86-21-5423-7435 (Q.J.)
}

Academic Editor: Paul B. Tchounwou

Received: 10 September 2016; Accepted: 14 November 2016; Published: 17 November 2016

\begin{abstract}
Background: Culex pipiens molestus was first reported in Shanghai in 2010. The population structures and seasonal distributions of Culex pipiens subspecies C. p. molestus, Culex pipiens pallens, and Culex pipiens quinquefasciatus are not well known. Methods: From late February to November 2013, we conducted daily field surveillance of mosquitoes at eight sites at two green lands and three residential areas in downtown Shanghai. Morphological comparison and DV/D ratios (DV/D is an indicator of mosquito taxonomy) were used to identify adult mosquitoes. Results: The distribution curves of the Culex pipiens complex members indicated seasonal fluctuations. The temperature range of $20-25^{\circ} \mathrm{C}$ was the most suitable for adult activity. Micro-environmental factors may differentiate the complex population structures. Hybridization between C. p. pallens and C. p. quinquefasciatus was common and neither " $\mathrm{DV} / \mathrm{D}=0.40$ " nor " $\mathrm{DV} / \mathrm{D}=0.50$ " can distinguish these subspecies and their hybrids. Conclusion: the population structure of the Culex pipiens complex is complex and characterized by significant hybridization. Measures other than DV/D ratios are needed for the discrimination of subspecies. The C. p. molestus invasion might result in the transmission of novel vector-borne diseases in Shanghai.
\end{abstract}

Keywords: Culex pipiens complex; population structure; seasonality; micro-environment; DV/D ratio; C. p. molestus; downtown Shanghai

\section{Introduction}

The Culex pipiens complex (Diptera: Culicidae) has been extensively studied but the taxonomic composition of the group remains unclear [1,2]. Vinogradova [1] considered the complex to include: C. p. pipiens with the typical, anautogenous pipiens form (form C. p. pipiens) as well as the autogenous molestus form (form C. p. molestus), C. p. quinquefasciatus, C. p. pallens, C. torrentium and C. vagans. The C. pipiens complex, according to Sirivanakam (1976), mainly includes the four forms C. p. pipiens, C. p. molestus, C. p. pallens and C. p. quinquefasciatus [3].

These latter four members of the Culex pipiens complex have been recorded in China (Figure 1) [4]. Two types of ubiquitous "house mosquitoes" (C. p. quinquefasciatus and C. $p$. pallens) are widely distributed in southern and northern China, respectively, with intermediates of the two found in a zone approximately between the $30^{\circ}$ and $32^{\circ} \mathrm{N}$ latitude [5]. The typical C. p. pipiens is only found in the Xinjiang Uygur autonomous region, and autogenous C. p. molestus was first reported in China in the Beijing underground water system in 1992 [6]. Up until now, only five regions in China have reported the presence of C. p. molestus, including Beijing (1993) [6], Shenyang of Liaoning Province [7], Manzhouli of 
the Inner Mongolia autonomous region [8], Wuhan of Hubei Province [9] and Shanghai [10]. Shanghai is located between $30^{\circ} 40^{\prime}$ and $31^{\circ} 53^{\prime} \mathrm{N}$, the overlap area of C. p. quinquefasciatus and C. p. pallens, so both the forms and their hybrids are present in the region. Since C. p. quinquefasciatus and C. p. pallens females are morphologically undistinguishable, the two forms as well as their hybrids usually are classified as C. p. pallens. Therefore, the composition of the C. pipiens complex population in this area is still unclear. In Shanghai, the proportion of the autogenous C. p. molestus population is unknown. This subspecies was first reported by Ji-shuhong et al. in 2010, after captures were made in an underground garage catch basin [10]. Between 2013 and 2015, we also found several autogenous C. p. molestus breeding in the underground catch basins of downtown Shanghai.

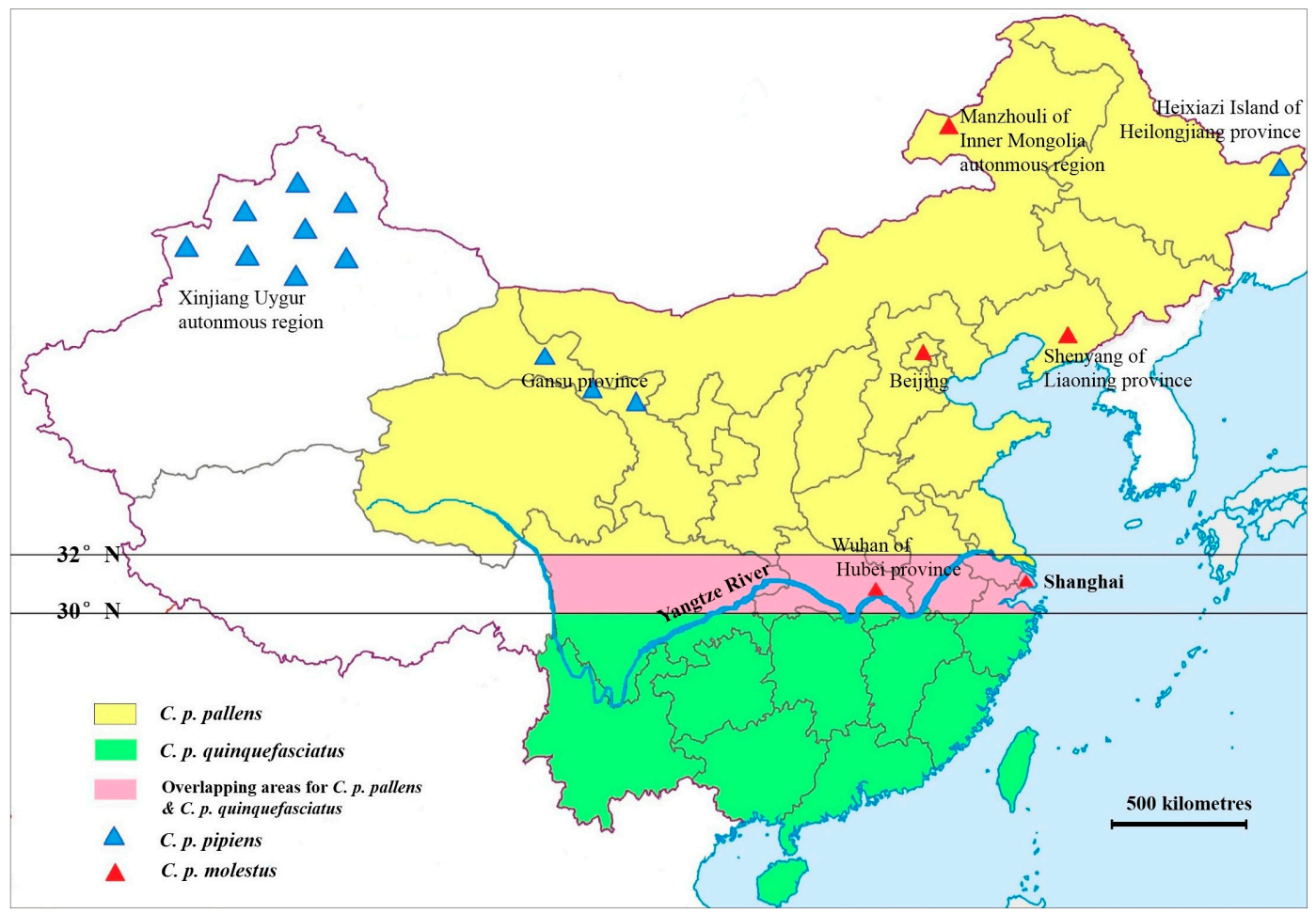

Figure 1. Approximate distribution of Culex pipiens complex mosquitoes in China (map constructed mainly from References [5-10]).

The C. pipiens complex plays an important role in the transmission of several pathogens including West Nile virus (WNV), St. Louis encephalitis virus, and filarial worms [11-13] as well as wildlife pathogens such as avian malaria (Plasmodium spp.) [14]. In the past two decades, the presence of C. pipiens in urban areas has attracted considerable attention due to their role as the major vector of WNV in temperate regions such as Europe and North America [15-18].

In China, members of the C. pipiens complex have been important vectors of Wuchereria bancrofti [4]. In North America, C. pipiens is strongly associated with West Nile virus transmission. Most studies on the $C$. pipiens complex have focused on species composition or population structure, while their temporal distributions are ignored. Information about the population structure, and spatial and temporal distributions of the different C. pipiens subspecies, biotypes or the hybrids is lacking in most areas of China due to the lack of sensitive and specific high-throughput screening of large sample sizes. To provide a rationale for WNV preventions in Shanghai, we developed a detailed investigation on the population structure and distribution of the C. pipiens complex, as well as their variances caused by micro-environmental factors. 


\section{Materials and Methods}

\subsection{Locations and Characteristics of Study Areas}

Fieldwork was carried out at the central area of Shanghai Municipality, located in east China $\left(31^{\circ} 13^{\prime} \mathrm{N}, 121^{\circ} 27^{\prime} \mathrm{E}\right.$ and $3.5 \mathrm{~m}$ above sea level), at two green lands (People's Park and People's Square) and three different residential environments. People's Park and People's Square are located in the center of Shanghai. The area is surrounded by busy roads, and the busiest People's Square Subway Station The exchange center for the subway Lines 1, 2, and 8 is located underground.

Mosquito surveillance sites 1-3 were set in People's Park located south of Nanjing Lu Road. The sites represented the west, middle and east area. The east and west parts have plentiful tall-vegetation and landscape architectures, including pavilions, corridors, artificial hills, lotus pond and pergolas, tourists can have a rest, play chess, or have a picnic on the stone tables and stone stools in the jungle of the area. With less vegetation, a small children's playground with several amusement facilities, a teahouse, an outdoor theater, and a dance hall can be found in the vast middle area. Two mosquito surveillance sites (sites 4 and 5) were set at the east and west areas of the People's Square. There is an artificial dovecot in the west part of the area, thousands of doves are active in this part. Three mosquito surveillance sites (sites 6-8) were located in residential areas in downtown Shanghai (Figure 2 and Table 1). Daily meteorological data was obtained from Shanghai Meteorological Center.

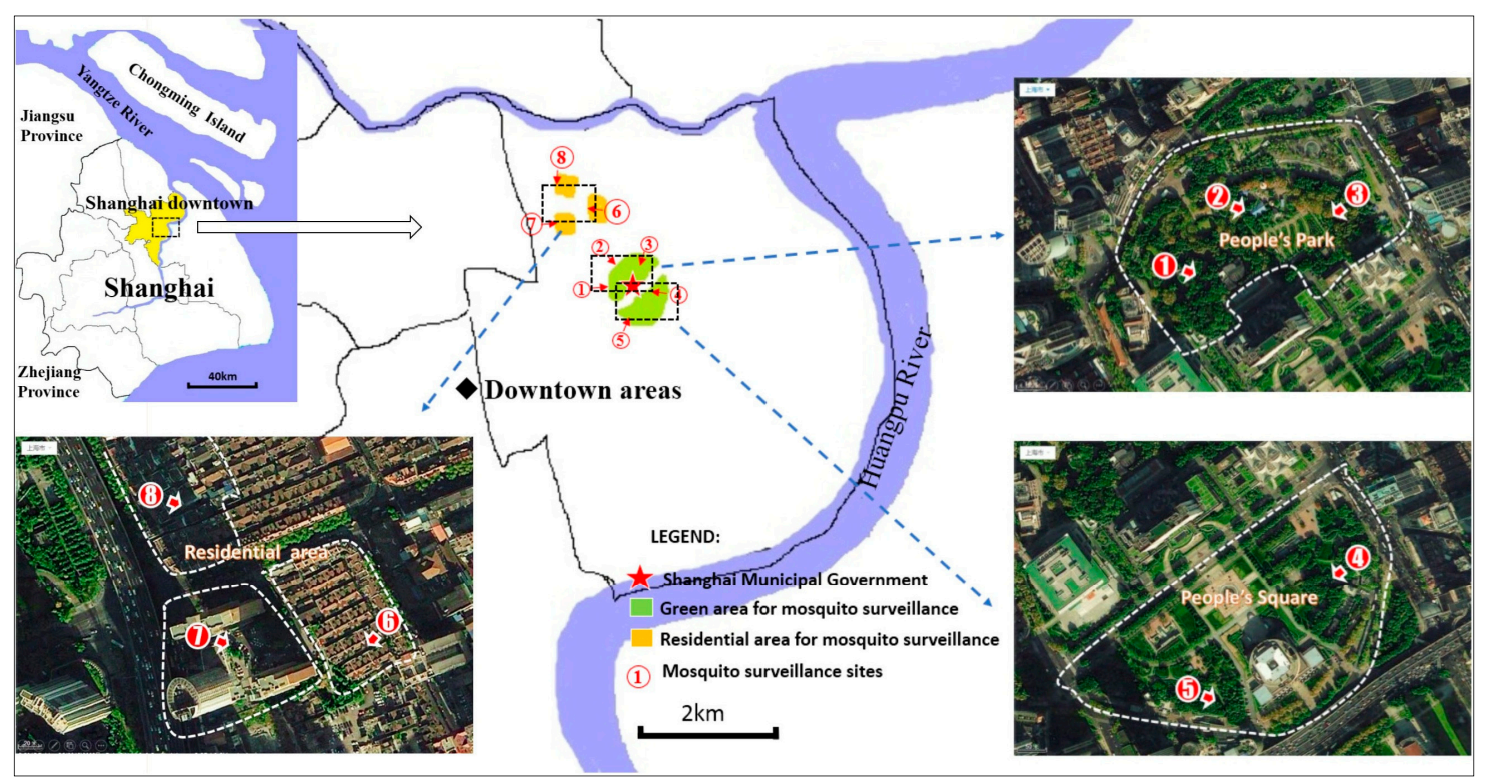

Figure 2. Regions and sites for mosquito surveillance in downtown Shanghai (Huangpu district).

Table 1. Geographical information of the eight mosquito surveillance sites.

\begin{tabular}{cccc}
\hline Site Numbers & Area & Environmental Types & Coordinates \\
\hline 1 & People's Park & Green land (Park) & $31^{\circ} 13^{\prime} 59.72^{\prime \prime} \mathrm{N} 121^{\circ} 28^{\prime} 02.06^{\prime \prime} \mathrm{E}$ \\
2 & People's Park & Green land (Park) & $31^{\circ} 14^{\prime} 03.10^{\prime \prime} \mathrm{N} 121^{\circ} 28^{\prime} 06.26^{\prime \prime} \mathrm{E}$ \\
3 & People's Park & Green land (Park) & $31^{\circ} 14^{\prime} 02.48^{\prime \prime} \mathrm{N} 121^{\circ} 28^{\prime} 13.02^{\prime \prime} \mathrm{E}$ \\
4 & People's Square & Green land & $31^{\circ} 13^{\prime} 53.01^{\prime \prime} \mathrm{N} 121^{\circ} 28^{\prime} 19.12^{\prime \prime} \mathrm{E}$ \\
5 & People's Square & Green land & $31^{\circ} 13^{\prime} 47.13^{\prime \prime} \mathrm{N} 121^{\circ} 28^{\prime} 09.01^{\prime \prime} \mathrm{E}$ \\
6 & Residential area & Old types “Shikumen" & $31^{\circ} 14^{\prime} 11.15^{\prime \prime} \mathrm{N} 121^{\circ} 27^{\prime} 48.74^{\prime \prime} \mathrm{E}$ \\
7 & Residential area & High-rise residential & $31^{\circ} 14^{\prime} 11.18^{\prime \prime} \mathrm{N} 121^{\circ} 27^{\prime} 45.45^{\prime \prime} \mathrm{E}$ \\
8 & Residential area & Old Villa & $31^{\circ} 14^{\prime} 15.83^{\prime \prime} \mathrm{N} 121^{\circ} 27^{\prime} 42.76^{\prime \prime} \mathrm{E}$ \\
\hline
\end{tabular}




\subsection{Study Design}

From late February to November 2013 (224 days), adult mosquito surveillance occurred daily at the eight aboveground sites (Figure 2). Mosquitoes were captured using a $\mathrm{CO}_{2}$-light trap and preserved in $75 \%$ ethanol. Compressed $\mathrm{CO}_{2}$ gas $(5 \mathrm{~kg} /$ cylinder) and trapping-bait (product simulating body secretions, produced by Bat King, Shanghai, China) were replaced every five and 20 days, respectively.

\subsection{Identification and Classification of Captured Mosquitoes}

With reduced thoracic chaetotaxy (the presence of specific setae and/or scales), wild-caught females of C. pipiens complex subspecies were morphologically indistinguishable [19] and were discarded. Adult males were dissected and classified according to the morphological characters of their genitalia. There are four forms of $C$. pipiens complex in downtown Shanghai. Phallosome differences were apparent among typical C. p. molestus, C. p. quinquefasciatus and C. p. pallens, though there was a small percentage of atypical species as well as putative hybrids. We considered the typical and atypical subtypes as one separate form and the hybrids as another separate form.

DV is the extension of the ventral arm laterally of its intersection with the dorsal arm and D is the distance between the two intersections of the dorsal and ventral arms. Calculation of the DV/D ratio is done according to Barr [20]. Median, "Mean \pm STD (standard deviation)" and interval from minimum to maximum were the indicators used to describe the distribution and dispersion of DV/D ratio among the four C. pipiens forms. DV measurements were recorded and their means calculated. Measurements were made by using an ocular micrometer at 100 diameters magnification, and were estimated to the nearest half of a scale division.

\subsection{Statistical Analysis}

Data were analyzed using the SPSS version 11.5 (SPSS Inc., Chicago, IL, USA) statistical package. The relative abundance of mosquito forms was expressed as "mosquito counts" instead of "mosquito density" because the size of male samples was relatively small. The variance between the proportions of different forms of C. pipiens complex was tested by chi-square analysis. Differences of DV/D values among the four forms were compared by nonparametric analysis of variance (Kruskal-Wallis test) and the Nemenyi test for post hoc testing.

\section{Results}

\subsection{Population Structure}

A total of 23,919 adult mosquitoes were captured at the eight sampling sites, and the collections included four mosquito species predominated by the C. pipiens complex ( $n=12,334 ; 51.57 \%$ of total); the other three species were Aedes albopictus, Culex tritaeniorhynchus and Anopheles sinensis. A total of 735 male Culex pipiens complex mosquitoes (5.96\%) were dissected, of which 721 (5.85\%) were morphologically identical to their type specimens of subspecies or forms (Figure 3).

Among the four forms, C. p. quinquefasciatus $(n=274,38.00 \%)$ and C. p. molestus ( $n=223,30.93 \%)$ were the most dominant subspecies; both were significantly more than C. p. pallens ( $n=135,18.72 \%)$ $\left(38.00 \%\right.$ vs. $18.72 \%, \chi^{2}=28.775, p<0.001 ; 30.93 \%$ vs. $\left.18.72 \%, \chi^{2}=65.943, p<0.001\right)$. The hybrid form of pallens and quinquefasciatus was captured in lesser numbers $(n=89,12.34 \%)$, and the ratio of the four forms was approximately 4:3:2:1 (Table 2). The population structure varies among surveillance sites. C. p. molestus was abundant in sites 3-5 (52.56\%, $43.21 \%$ and $54.00 \%$, respectively), and was much less common in the other five sites, especially the residential area sites $6-8(8.75 \%, 16.00 \%$ and $9.72 \%$, respectively). The distribution of C.p. quinquefasciatus and C. p. pallens was relatively similar among the eight sites, although slightly less in sites 3-5 in contrast to the high proportion of C. p. molestus (Figure 4a).

Differences in micro-environments might have influenced the population structure of the C. pipiens complex. Sites 1-3 at People's Park are examples: even though they were at nearby locations within 
the same park, the three sites displayed distinct population structures (see Figure 4a). The mosquito population size in site 3 was much greater than at sites 1 and $2\left(n=215\right.$ vs. $n=28, \chi^{2}=223.561$, $p<0.001 ; n=215$ vs. $\left.n=98, \chi^{2}=80.832, p<0.001\right)$; second, the proportion of $C$. $p$. molestus at site 3 was much larger than those on sites 1 and 2 (52.56\% vs. $17.86 \%, \chi^{2}=11.942, p=0.001 ; 52.56 \%$ vs. $18.37 \%$, $\left.\chi^{2}=32.336, p<0.001\right)$. Finally, the proportion of C. p. pallens and C. $p$. quinquefasciatus in the three sites differed (Table 2).

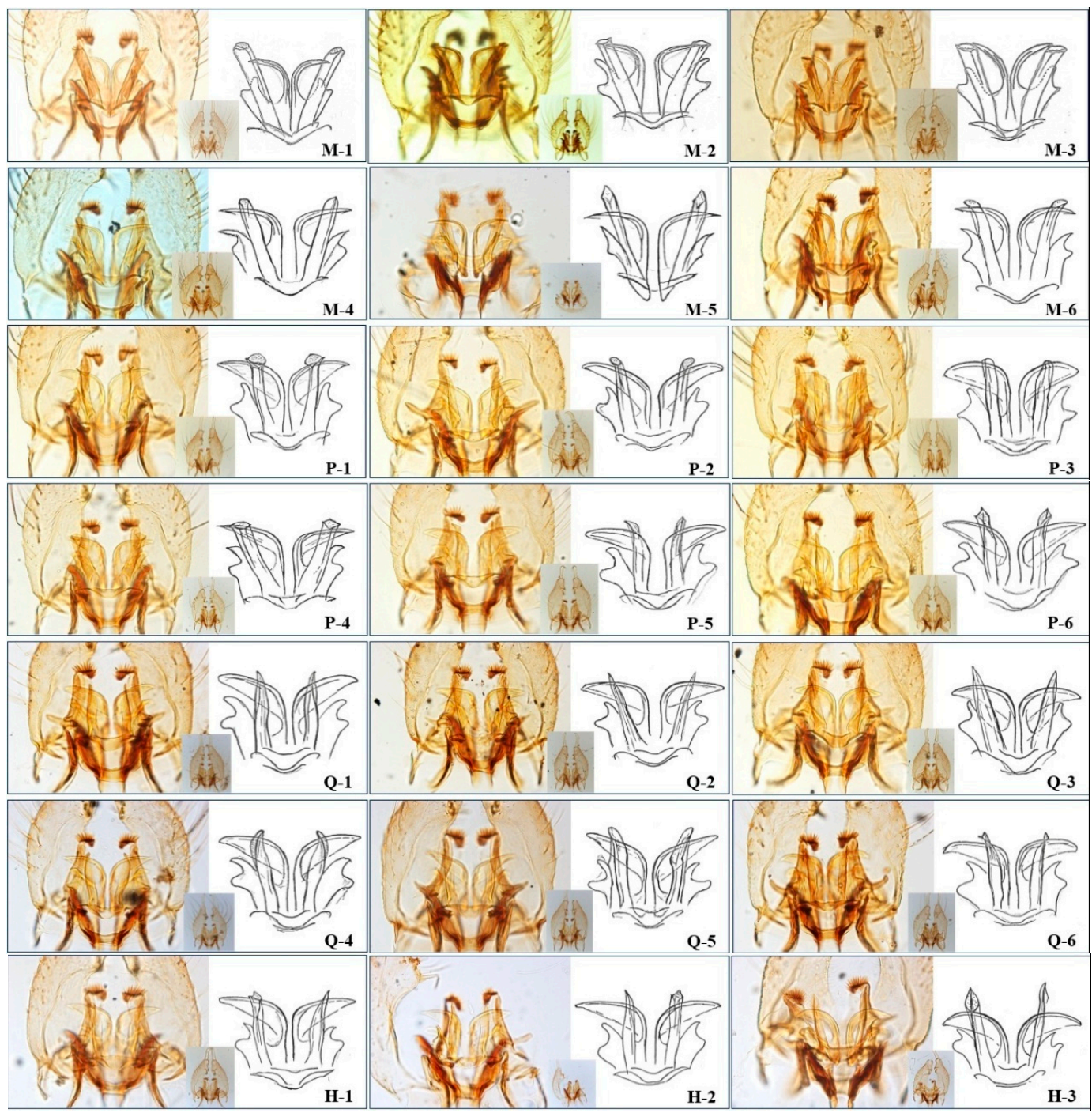

Figure 3. Genitalia (dorsal view, post-rotation) of male Culex pipiens complex samples collected in downtown Shanghai, China. Typical C. p. molestus (M-1, 2, 3): the dorsal arms are divergent and broad, and their tips are thick and blunt, the ventral arms are narrow, short and sharply bent laterally. Atypical C. p. molestus (M-4, 5, 6): the ventral arms are longer than the typical ones, and the DV/D ratio increases accordingly. Typical C. p. pallens (P-1, 2, 3): the dorsal arms are divergent and broad, and their tips are thick and bluntly round (or truncate), the ventral arms are broad, long and extend caudolaterally (the same as C. p. quinquefasciatus). Atypical C. p. pallens (P-4, 5, 6): the ventral arms are narrower (P-4), or the cross-section of the dorsal arms' tips are irregular (P-5, 6). Typical C. p. quinquefasciatus (Q-1, 2, 3 ): the dorsal arms are parallel (or divergent, Q-2,3) and narrow, and their tips are markedly pointed, the ventral arms are broad, long and extend caudolaterally. Atypical C. p. quinquefasciatus (P-4, 5, 6): the tips of the dorsal arms are "thumb-shaped" (Q-4), or the dorsal arms are a little bit thicker (Q-5) or flat (Q-6). Hybrids of pallens and quinquefasciatus (H-1, 2, 3): morphological character of the dorsal arms is between C. p. pallens and C. p. quinquefasciatus. 
Table 2. Composition and distribution (Counts/pcs, proportion/\%) of Culex pipiens complex mosquitoes in downtown Shanghai.

\begin{tabular}{cccccc}
\hline Site Numbers & C. p. molestus & C. p. pallens & $\begin{array}{c}\text { Hybrids of pallens \& } \\
\text { quinquefasciatus }\end{array}$ & $\begin{array}{c}\text { C. } \boldsymbol{p} \text {. } \\
\text { quinquefasciatus }\end{array}$ & Sum \\
\hline 1 & $5(17.86)$ & $10(35.71)$ & $2(7.14)$ & $11(39.29)$ & 28 \\
2 & $18(18.37)$ & $21(21.43)$ & $16(16.33)$ & $43(43.88)$ & 98 \\
3 & $113(52.56)$ & $22(10.23)$ & $17(7.91)$ & $63(29.30)$ & 215 \\
4 & $35(43.21)$ & $11(13.58)$ & $6(7.41)$ & $29(35.80)$ & 81 \\
5 & $27(54.00)$ & $6(12.00)$ & $3(6.00)$ & $14(28.00)$ & 50 \\
6 & $7(8.75)$ & $19(23.75)$ & $14(17.50)$ & $40(50.00)$ & 80 \\
7 & $4(16.00)$ & $7(28.00)$ & $6(24.00)$ & $8(32.00)$ & 25 \\
8 & $14(9.72)$ & $39(27.08)$ & $25(17.36)$ & $66(45.83)$ & 144 \\
People's Park (1-3) & $136(39.88)$ & $53(15.54)$ & $35(10.26)$ & $117(34.31)$ & 341 \\
People's Square (4,5) & $62(47.33)$ & $17(12.98)$ & $9(6.87)$ & $43(32.82)$ & 131 \\
Residential (6-8) & $25(10.04)$ & $65(26.10)$ & $45(18.07)$ & $114(45.78)$ & 249 \\
Total & $223(30.93)$ & $135(18.72)$ & $89(12.34)$ & $274(38.00)$ & 721 \\
\hline
\end{tabular}
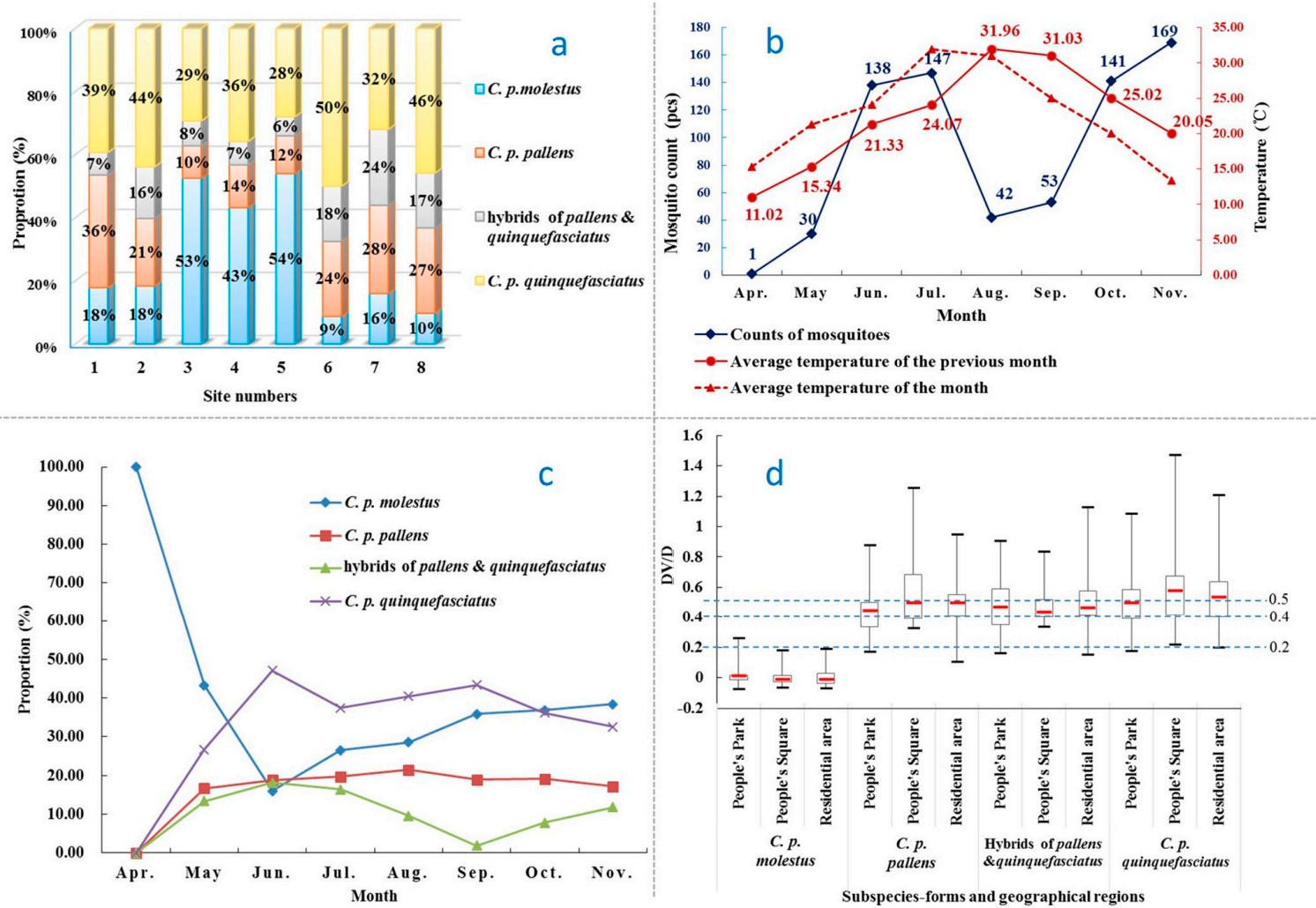

Figure 4. Characteristics of the structure, spatial and temporal distribution of Culex pipiens complex in downtown Shanghai, China. (a) Proportion of the four forms of Culex pipiens complex at the eight sites in downtown Shanghai; (b) Correlation between temperature and counts of Culex pipiens complex mosquitoes in downtown Shanghai; (c) Seasonal fluctuation of Culex pipiens complex members in downtown Shanghai; (d) DV/D ratio of Culex pipiens complex members in downtown Shanghai.

\subsection{Seasonal Fluctuation}

Mosquito counts increased rapidly as mean daily temperatures rose. Counts peaked in June and July ( $n=138$ and 147). As the temperature continued to rise in August and September, mosquito counts declined sharply ( $n=42$ and 53), and then peaked again in October and November $(n=141$ and 169) as the temperatures declined. There existed an obvious relationship between temperature and the mosquito density, but the impact of the temperature on mosquitoes had an approximate one-month 
lag time. The most suitable temperature interval for the C. pipiens complex ranged between 20 and $25{ }^{\circ} \mathrm{C}$ (Figure $4 \mathrm{~b}$ ).

The proportion of $C$. pipiens complex members also exhibited distinct seasonal variation. During the low-temperature months (April, May, and November), C. p. molestus dominated the complex composition and peaked in November $(n=65$, Proportion $=38.46 \%)$. In contrast, $C$. $p$. quinquefasciatus dominated during the high-temperature months (June to September), and peaked in June ( $n=65$, proportion $=47.10 \%$ ). Population levels of $C$. $p$. pallens were relatively stable during the study period (Figure 4c).

\subsection{DV/D Ratio}

The DV/D ratio of C. p. molestus was the smallest among the four subspecies ( -0.012 vs. 0.479 , 0.469 , and 0.500 for median; $p<0.01$ ); however, its maximum of 0.258 also exceeded 0.20 , which is the criteria for the classification of C.p. pipiens or molestus. Although the DV/D ratio of C.p. pallens was significantly smaller than that of C. p. quinquefasciatus ( 0.479 vs. 0.500 of median, Nemenyi test, $p=0.04)$, the difference was quite small. There was no significant difference between hybrids and C. p. pallens or C. p. quinquefasicatus (Table 3).

Table 3. DV/D ratios of Culex pipiens complex members in Shanghai downtown, China.

\begin{tabular}{ccccc}
\hline Forms & $\boldsymbol{n}$ & Median & Mean \pm STD & Intervals \\
\hline C. p. molestus & 223 & $-0.012^{\mathrm{a}}$ & $0.004 \pm 0.048$ & $-0.079-0.258$ \\
C. $p$. pallens & 135 & $0.479^{\mathrm{b}}$ & $0.486 \pm 0.168$ & $0.100-1.250$ \\
Hybrids of pallens and quinquefasciatus & 89 & $0.469^{\mathrm{b}, \mathrm{c}}$ & $0.490 \pm 0.180$ & $0.150-1.125$ \\
C. $p$. quinquefasciatus $^{\mathrm{c}}$ & 274 & $0.500^{\mathrm{c}}$ & $0.539 \pm 0.195$ & $0.171-1.467$ \\
\hline
\end{tabular}

$\mathrm{a}, \mathrm{b}, \mathrm{c}$ : Nemenyi test for post hoc comparison between groups, no significant difference between the same letters, but there are significant differences between different letters, like ${ }^{\mathrm{a}}$ and ${ }^{\mathrm{b}}, \mathrm{b}$ and ${ }^{\mathrm{c}}$, as well as ${ }^{\mathrm{a}}$ and ${ }^{\mathrm{c}}, p<0.05$.

The median value and most of C. p. molestus' DV/D ratios were below 0.2; however, neither 0.4 nor 0.5 could accurately separate the forms of $C$. p. pallens, C. p. quinquefasciatus and the hybrids (Figure 4d).

\section{Discussion}

The distribution of the C. pipiens complex, and in particular of the C. p. molestus subspecies, is of great importance in Shanghai as well as in the rest of China because of the increased number of underground mosquito breeding places in towns [1]. In Shanghai, C. p. molestus had never been reported before 2010, when Ji-shuhong et al. first reported the autogenous form of C. pipiens underground in the Yangpu district [10]. Until recently, only five regions in China had reported the presence of C. p. molestus. This subspecies was mostly found breeding in underground environments. Shanghai appears to be the only place in China where C. p. molestus is breeding in both half-closed underground garages and the open aboveground environment [10]. In a recent study (2013 to 2015), we confirmed that thousands of autogenous C. pipiens larvae existed in catch basins of several underground garages and, C. p. molestus larvae bred in an aboveground surface sewer of People's Square (unpublished). Unfortunately, data on C. pipiens dispersal are limited. Most documents relate to C. p. quinquefasciatus which was probably introduced to the Hawaii Islands in the 18th century [21]. C. $p$. quinquefasciatus is easily transported by aircraft or boats whereas, under similar conditions, C. p. molestus is much rarer. Vinogradova [1] inferred that the chances for a successive introduction of C. p. molestus are small in the temperate belt, since (i) their manner of reproduction in closed underground habitats makes it unlikely that they will be transported by a vehicle and (ii) climatic conditions restrict the periods when mosquitoes can disperse from their habitats. If C. p. molestus mosquito transportation does occur, the female(s) could encounter difficulties in finding suitable 
underground habitats for oviposition in the new environment. Waves of founder mosquitoes are likely to be repeatedly transported before a new stable local population is established.

Generally, autogenous C. p. molestus is associated with breeding sites that provide only limited access and egress for these insects [22]. However, the status of C. p. molestus in downtown Shanghai appears to differ from the norm. Prior to this study, we estimated that there would be few adult C. $p$. molestus active aboveground and these would originate from the abundant underground garages or urban basements of Shanghai. However, the proportion of C. p. molestus in the open environment was as high as $52.56 \%$ (site 2). One explanation is that C. p. molestus has now adapted to the urban aboveground environment in Shanghai. While thousands of adult C. p. molestus leave their underground habitats and return to the underground for oviposition, a small proportion may have located suitable habitats for oviposition aboveground. This was verified when we found C. p. molestus larvae in an aboveground surface sewer of People's Square. Regardless of the types of habitats they would prefer for oviposition, a high proportion of adult C. p. molestus in the open environment would minimize the dispersal constraints mentioned above, and greatly facilitate dispersal. C. p. molestus in Shanghai could soon be introduced to the surrounding areas or more distant regions by vehicles, boats and aircraft. An alternative interpretation is that the form C. p. molestus in our study is actually the form C. p. pipiens, which is anautogenous, eurygamous (only mating in outdoor swarms) and capable of diapause $[19,23]$. Vinogradova noted that both forms occur in sympatry almost throughout the entire area of distribution. In this study, if specimens of the form C. p. pipiens are included, they could not be separated from C. p. molestus morphologically in this study, and an appropriate molecular approach may be needed for further analysis [24]. In any event, the proportion of the form C. p. molestus (or the form C. p. pipiens) is fairly high in the aboveground environment. This is not good news for either Shanghai or for China because it could greatly increase the risk of transmission of WNV and other diseases vectored by the C. pipiens complex.

C. pipiens complex mosquitoes are difficult to classify by simple methods and many forms are routinely determined to be C. $p$. pallens. This is usually inaccurate, since the proportion of C. p. pallens is smaller than both C. p. quinquefasciatus and C. p. molestus. In addition, there is often a proportion of hybrid forms. As an approach for morphological classification, the DV/D ratio was developed from the $\mathrm{D} / \mathrm{V}$ ratio. The latter is defined as a ratio of distances between the dorsal and ventral arms, which is also a method of distinguishing individuals of $C$. pipiens that was suggested by Sundararaman (1949) [25] and later modified by Barr (1957) [20] to give "somewhat more objective measurements". The method has since been improved to result in the well-known $\mathrm{DV} / \mathrm{D}$ ratio. The DV/D ratio remains the most reliable method for distinguishing adult C. p. pipiens and C. p. quinquefasciatus (" $<0.20 "=$ pipiens) [26]. In this study, there was no need to calculate DV /D ratios to separate C. p. pipiens and C. p. quinquefasciatus, since the dorsal and ventral arms and lateral plate are distinctive enough to distinguish the males of the two subspecies. The DV/D ratio is useful for distinguishing parental and hybrid forms in zones of introgression and establishing the temporal and geographic extent of hybridization. It works well in the United States for differentiating the subspecies and for detecting the presence of hybrids [20,27]. The DV/D ratio was also used to establish the existence of a continuous, self-maintaining intermediate population in Memphis, Tennessee [28]. Barr suggested that 0.40 should be the criteria to separate C. p. quinquefasciatus from other subspecies (" $>0.40 "$ = quinquefasciatus), but Jakob et al. suggested that 0.50 should be the lowest DV/D value denoting specimens as quinquefasciatus [26]. Researchers in China and other Asian countries suggest that the DV/D ratio is a poor indicator for mosquito classification, since the values of DV/D vary greatly according to latitude, season, and even use of the technique [29-32]. In this study, neither $\mathrm{DV} / \mathrm{D}=0.40$ nor $\mathrm{DV} / \mathrm{D}=0.50$ provided a good criterion to differentiate $C$. $p$. quinquefasciatus and C. p. pallens or the hybrids, since the values of DV/D among the three forms overlapped in the $0.40-0.50$ interval. The dorsal arms of most C. p. quinquefasciatus are divergent with tips pointed, which is different from the classical definition of "parallel and pointed", and greatly different from the atypical or hybrid examples. We therefore classified both the "divergent and pointed" and "parallel 
and pointed" forms as typical C. p. quinquefasciatus. Divergent dorsal arms resulted in relatively low $\mathrm{DV} / \mathrm{D}$ ratio values. The median value of the DV/D ratio of C. p. quinquefasciatus in People's Park was 0.50 , and the mean DV/D ratio was 0.539 .

Urbanization is one of the major human activities impacting biodiversity. Human intervention creates highly heterogeneous environments for invertebrates, in which many biotic and abiotic factors could affect the community structure, impacting species richness and the species composition [33]. These factors may occur at different spatial scales, from the macro-environment to the micro-environment [34-36]. Eight field sites for mosquito surveillance, with the average distance of less than $600 \mathrm{~m}$ between each other, were micro-environments within the urban area. The effect of the micro-environment on the richness (density) and the complex composition (population structure) is obvious in this study, especially for C. p. molestus. The factors influencing the mosquito population structure and density include types of adult mosquito habitats, types and number of breeding sites, and blood meal sources. In our understanding, the most important factor might be the types and numbers of breeding sites. Generally, C. p. molestus prefer breeding sites that provide only limited access and egress such as underground garages or basements. However, the eight sites are all surrounded with tall buildings that have many underground garages in the urban center. A plausible explanation is that C. p. molestus can oviposite in at least one type of aboveground breeding site, and such breeding sites are widely distributed at sites 3-5. The finding of an aboveground breeding site in People's Square during the summer of 2015 supports this hypothesis.

Seasonal progressions and resulting changes (e.g., temperature and photo period) are other essential external factors greatly affecting mosquito population dynamics. In the relatively cold April, only male C. p. molestus were captured. This is not difficult to interpret since C. p. molestus can survive and breed underground without diapause while development of other forms decreases dramatically during the winter with significant activity only resuming in the spring. C. p. quinquefasciatus also forgoes diapause [37], but its ability to adapt to both low temperature and underground habitats is apparently less than C. p. molestus. The density of the C. pipiens complex is closely related to the temperature, but the effect of temperature is delayed for approximately one month. This lag phase can provide an excellent time window for mosquito control.

\section{Conclusions}

The population structure of Culex pipiens in Shanghai is complex and includes a significant amount of hybridization. C. p. quinquefasciatus, C. p. pallens and C. p. molestus coexist in Shanghai, producing a fairly high proportion of hybrids of pallens and quinquefasciatus and many morphologically atypical forms of the C. pipiens complex. In Shanghai, the C. p. molestus population is increasing and its habitats are no longer confined to the underground areas. The increasing number of C. p. molestus and its adaptation to aboveground habitats may facilitate its dispersal to other areas around China and a larger population might result in the transmission of novel vector-borne diseases. The DV/D ratio could not be used to differentiate the C. pipiens complex mosquitoes in Shanghai, since the values of the DV/D ratio overlapped in the three forms of the complex. The most practical method for classification is based on the morphological characters of male genitalia. The micro-environment and temperature can significantly affect the complex population structures.

Acknowledgments: We thank Shu-Hong Ji (Center for Disease control and prevention in Yangpu District) and Yi-Bin Zhou (Shanghai Municipal Center for Disease control and prevention) for technical support in making mosquito genitalia slides and classification. We thank LetPub (www.letpub.com) for its linguistic assistance during the preparation of this manuscript.

Author Contributions: Qingwu Jiang, Jianjun Zhou, Chenglong Xiong, and Qiang Gao conceived the study and drafted the manuscript. Jianjun Zhou and Fei Su designed and led the field work and collections. Qiang Gao and Chenglong Xiong conducted the experiments, analyzed the data and wrote the manuscript. Hui Cao designed the field work and collected samples. All authors have read and approved the final manuscript.

Conflicts of Interest: The authors declare that they have no competing interests. 


\section{References}

1. Vinogradova, E.B. Culex pipiens Pipiens Mosquitoes: Taxonomy, Distribution, Ecology, Physiology, Genetics, Applied Importance and Control; Pensoft: Sofia, Bulgaria, 2000; pp. 1-25.

2. Harbach, R.E. Culex pipiens: Species versus species complex taxonomic history and perspective. J. Am. Mosq. Control Assoc. 2012, 28, 10-23. [CrossRef] [PubMed]

3. Sirivanakam, S. Medical entomology studies-III. A revision of the subgenus Culex in the Oriental Region (Diptera: Culicidae). Contrib. Am. Entomol. Inst. (Ann Arbor) 1976, 12, 1-272.

4. Zhao, T.; Lu, B. Biosystematics of Culex pipiens complex in China. Insect Sci. 1995, 2, 1-8. [CrossRef]

5. Lu, B. Fauna Sinica. Insect Vol. 8, Diptera: Culicidae 1; Science Press: Beijing, China, 1997; pp. 5-410.

6. Zhao, T.; Lu, B. A new record of Culex pipiens molestus in China and Studies of autogeny and systematics. Chin. J. Vector Biol. Control 1993, 4, 241-243.

7. Song, S.; Zhao, T.; Dong, Y.; Lu, B. Hybridization and Wolbachia infection in the Culex pipiens complex in China. Acta Entomol. Sin. 2001, 45, 706-710.

8. Xing, D.; Zhao, T. A new record of Culex pipiens mosquito molestus in Inner Mongolia and its infection with Wolbachia. Acta Parasitol. Med. Entomol. Sin. 2013, 20, 251-254.

9. Wu, T.P.; Hu, Q.; Zhao, T.; Tian, J.H.; Xue, R.D. Morphological studies on Culex molestus of the Culex pipiens complex (Diptera: Culicidae) in underground parking lots in Wuhan, central China. Fla. Entomol. 2014, 97, 1191-1198. [CrossRef]

10. Ji, S.; Zhao, T.; Leng, P.; Huang, H.; Mu, D.; Wang, J. A new record of Culex pipiens molestus in Shanghai and studies of autogeny and resistance to four insecticides. Acta Parasitol. Med. Entomol. Sin. 2010, 17, 170-173.

11. Reisen, W.K.; Milby, M.M.; Presser, S.B.; Hardy, J.L. Ecology of mosquitoes and St. Louis encephalitis virus in the Los Angeles Basin of California, 1987-1990. J. Med. Entomol. 1992, 29, 582-598. [PubMed]

12. Bogh, C.; Pedersen, E.M.; Mukoko, D.A.; Ouma, J.H. Permethrin-impregnated bednet effects on resting and feeding behaviour of lymphatic filariasis vector mosquitoes in Kenya. Med. Vet. Entomol. 1998, 12, 52-59. [CrossRef] [PubMed]

13. Turell, M.J.; Sardelis, M.R.; O'Guinn, M.L.; Dohm, D.J. Potential vectors of West Nile virus in North America. Curr. Top. Microbiol. Immunol. 2002, 267, 241-252. [PubMed]

14. Kimura, M.; Darbro, J.M.; Harrington, L.C. Avian malaria parasites share congeneric mosquito vectors. J. Parasitol. 2010, 96, 144-151. [CrossRef] [PubMed]

15. Hayes, E.B.; Komar, N.; Nasci, R.S.; Montgomery, S.P.; O’Leary, D.R.; Campbell, G.L. Epidemiology and transmission dynamics of West Nile virus disease. Emerg. Infect. Dis. 2005, 11, 1167-1173. [CrossRef] [PubMed]

16. Hubalek, Z. Mosquito-borne viruses in Europe. Parasitol. Res. 2008, 103, S29-S43. [CrossRef] [PubMed]

17. Zeller, H.; Lenglet, A.; Van Bortel, W. West Nile virus: The need to strengthen preparedness in Europe. Eurosurveillance 2010, 15, 1-2.

18. Andreadis, T.G. The contribution of Culex pipiens complex mosquitoes to transmission and persistence of West Nile virus in North America. J. Am. Mosq. Control Assoc. 2012, 28, S137-S151. [CrossRef] [PubMed]

19. Rudolf, M.; Czajka, C.; Borstler, J.; Melaun, C.; Jost, H.; von Thien, H.; Badusche, M.; Becker, N.; Schmidt-Chanasit, J.; Kruger, A.; et al. First nationwide surveillance of Culex pipiens complex and Culex torrentium mosquitoes demonstrated the presence of Culex pipiens biotype pipiens/molestus hybrids in Germany. PLoS ONE 2013, 8, e71832. [CrossRef] [PubMed]

20. Barr, A.R. The distribution of Culex p. pipiens and C. p. quinquefasciatus in North America. Am. J. Trop. Med. Hyg. 1957, 6, 153-165.

21. Van Dine, D.L. A sugarcane leafhopper in Hawaii. Hawaii Agric. Exp. Stn. Bull. 1904, 5, 29.

22. Spielman, A. Structure and seasonality of nearctic Culex pipiens populations. Ann. N. Y. Acad. Sci. 2001, 951, 220-234. [CrossRef] [PubMed]

23. Byrne, K.; Nichols, R.A. Culex pipiens in London underground tunnels: Differentiation between surface and subterranean populations. Heredity 1999, 82, 7-15. [CrossRef] [PubMed]

24. Bahnck, C.M.; Fonseca, D.M. Rapid assay to identify the two genetic forms of Culex (Culex) pipiens L. (Diptera: Culicidae) and hybrid populations. Am. J. Trop. Med. Hyg. 2006, 75, 251-255. [PubMed]

25. Sundararaman, S. Biometrical studies on intergradation in the genitaiia of certain populations of Culex pipiens and Culex quinquefasciatus in the United States. Am. J. Hyg. 1949, 60, 307-314. 
26. Jakob, W.L.; Francy, D.B. Observations on the DV/D ratio of male genitalia of Culex pipiens complex mosquitoes in the United States. Mosq. Syst. 1984, 16, 282-288.

27. Iltis, G.W. Biosystematics of the Culex pipiens Complex in Northern California. Ph.D. Thesis, University of California, Davis, CA, USA, 1966.

28. Jakob, W.L.; Daggers, S.A.; Francy, D.B.; Mullenix, J.; Moseley, K. The Culex pipiens complex in Memphis, Tennessee. Mosq. Syst. 1979, 11, 179-186.

29. Chen, H.; Lu, B. Study on the Culex pipiens complex in China. J. Guiyang Med. Univ. 1983, 8, 1-13.

30. Ishii, T. On the Culex pipiens group in Japan. III. A historical review of its research. IV. Review of the adult character (3). J. Sci. (Coll. Gen. Educ. Univ. Tokushima) 1980, 13, $26-62$.

31. Ishii, T. Integrated study onthe Culex pipiens complex: Species diversion in the Culex pipiens complex. Akaieka Newsl. 1991, 14, 5-40.

32. Zhao, T.; Lu, B. Biosystematic studies on the Culex pipiens complex of China: Mathematical analysis of DV/D ratio of male genitalia. Acta Entomol. Sin. 1994, 37, 446-449.

33. Rubio, A.; Bellocq, M.I.; Vezzani, D. Macro- and microenvironmental factors affecting tyre-breeding flies (Insecta: Diptera) in urbanised areas. Ecol. Entomol. 2013, 38, 303-314. [CrossRef]

34. Hansen, A.J.; Knight, R.L.; Marlzuff, J.M.; Powell, S.; Brown, K.; Gude, P.H. Effects of exurban development on biodiversity: Patterns, mechanisms, and research needs. Ecol. Appl. 2005, 15, 1893-1905. [CrossRef]

35. Paul, M.J.; Meyer, J.L. Streams in the urban landscape. Annu. Rev. Ecol. Syst. 2001, 32, 333-365. [CrossRef]

36. Rey, J.R.; Nishimura, N.; Wagner, B.; Braks, M.A.; O'Connell, S.M.; Lounibos, L.P. Habitat segregation of mosquito arbovirus vectors in south Florida. J. Med. Entomol. 2006, 43, 1134-1141. [CrossRef] [PubMed]

37. Wild, J.D.E. Photoperiodism in insects and mites. Annu. Rev. Enterp. 1962, 7, 1-26. [CrossRef]

(C) 2016 by the authors; licensee MDPI, Basel, Switzerland. This article is an open access article distributed under the terms and conditions of the Creative Commons Attribution (CC-BY) license (http:/ / creativecommons.org/licenses/by/4.0/). 